\title{
Operation and Performance of the CMS Electromagnetic Calorimeter
}

\author{
Martina Malberti* on behalf of the CMS Collaboration \\ Universitá \& INFN Milano Bicocca \\ E-mail: martina.malberti@cern.ch
}

The CMS Electromagnetic Calorimeter (ECAL) is a high resolution, finely grained calorimeter devised to measure photons and electrons at LHC. Built of lead tungstate crystals, it plays a crucial role in the search for new physics as well as in precision measurements in the Standard Model. The operation and general performance of the CMS ECAL in proton-proton collision at $\sqrt{s}=$ $7 \mathrm{TeV}$ are described. The precision of the inter-channel synchronization and calibration has been verified and improved exploiting in-situ data. Di-electron and di-photon states have been also used to verify and tune the energy scale. The quality of the offline data reconstruction, from low level quantities to showers, has been investigated and improved using known physics processes. Collision data and thorough data/Monte Carlo comparisons have been used to measure and tune the detector performance. First performance results are given.

Workshop on Discovery Physics at the LHC-Kruger 2010

December 05-10, 2010

Kruger National Park, Mpumalanga, South Africa

\footnotetext{
${ }^{*}$ Speaker.
} 


\section{Overview of the CMS electromagnetic calorimeter}

The Electromagnetic Calorimeter (ECAL) of the Compact Muon Solenoid (CMS) experiment [1] at the LHC [2] is a hermetic homogeneous calorimeter made of 75848 lead tungstate $\left(\mathrm{PbWO}_{4}\right)$ scintillating crystals. It consists of a central barrel region (EB) organized in 36 supermodules, each containing 1700 crystals, and two endcaps (EE) of 7324 crystals each. The scintillation light is readout by avalanche photodiodes (APDs) in the barrel and with vacuum phototriodes (VPTs) in the endcaps. Silicon preshower detectors are installed in front of the ECAL endcaps. The EB provides the coverage of pseudorapidity $|\eta|<1.479$ with EE extending to $|\eta|=3.0$. The ES covers $1.653<\eta<2.6$. The fine granularity and excellent energy resolution of the calorimeter have been optimized for the detection of the Higgs boson through its electromagnetic decay.

The ECAL energy resolution measured in electron test beams is parametrized as [1]

$$
\frac{\sigma(E)}{E}=\frac{2.8 \%}{\sqrt{E(G e V)}} \oplus \frac{12 \%}{E(G e V)} \oplus 0.3 \%
$$

for electrons incident on the center of crystals. The three contributions correspond to the stochastic, noise and constant terms. In the environment of CMS, for unconverted photons with energies above $100 \mathrm{GeV}$, the energy resolution is dominated by the constant term. As a consequence, the performance of the CMS ECAL at the LHC will depend mainly on the quality of its inter-calibration and monitoring. Achieving the design-goal inter-calibration precision of $0.5 \%$ in situ will be particularly important for a discovery of the Higgs boson in the decay channel $H \rightarrow \gamma \gamma$, one of the primary goals of the LHC physics program.

The status and stability of the CMS ECAL during LHC collisions in 2010 is presented and its calibration and performances discussed.

\section{ECAL status and stability}

During the first few months of the LHC collisions in 2010, the percentage of fully working channels in EB and EE is about $99.30 \%$ and $98.94 \%$, respectively. In ES, the percentage of fullyfunctional strips is $99.79 \%$ [3].

The stability of the entire system is crucial to achieve the goal constant term in the energy resolution. Among the different contributions to that are the temperature stability of the crystals and photodetectors and the crystal transparency, which can decrease with radiation. The temperature stability over two months has been measured to be about $0.0076^{\circ} \mathrm{C}$ and $0.015^{\circ} \mathrm{C}$ for $\mathrm{EB}$ and $\mathrm{EE}$ respectively [3]. These values are well within specifications, which allow for maximum variations of $0.05^{\circ} \mathrm{C}$ in $\mathrm{EB}$ and $0.1^{\circ} \mathrm{C}$ in EE. The light monitoring system itself shows a stability at the $0.03 \%$ level, which is much better than what is required to mantain the constant term in the ECAL energy resolution at the level of $0.5 \%$.

Figure 1 shows the energy spectra measured in individual EB channels from data and Monte Carlo (MC) simulation and the azimuthal distribution of the channel with the highest reconstructed energy. Variations as a function of $\phi$ reflect the modularity and inhomogeneity of the energy equivalent noise in ECAL. The excellent agreement between data and MC is due to the detailed simulation of the ECAL response and of energy equivalent noise in each channel. 

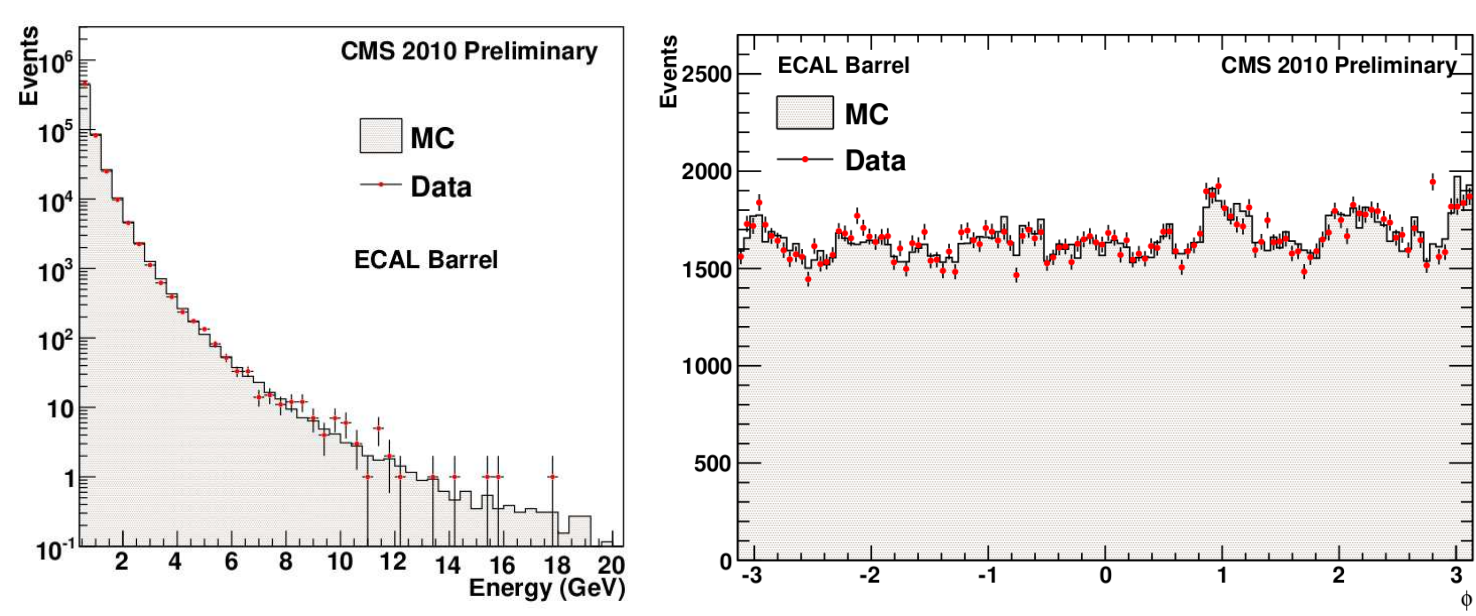

Figure 1: Single channel energy spectrum (left) and azimuthal distribution of the channel with the highest reconstructed energy (right) from $7 \mathrm{TeV}$ minimum bias collision events.

The precision time measurement is another important aspect of the ECAL performance, because it can be exploited in the rejection of backgrounds (such as cosmic rays, beam halo muons, electronic noise and out-of-time interactions) and for the identification of new particles (e.g. slow heavy charged R-hadrons). Collision data have been used to perform an in-situ synchronization of the channels and to study the timing performances, showing that a sub-nanosecond timing resolution is achievable by a single EB/EE channel for high energy deposits [3].

\section{ECAL calibration}

The ECAL calibration aims at the best estimate of the energy of electron and photons. The energy of electrons and photons spread over several crystals and can be expressed as

$$
E_{e, \gamma}=F_{e, \gamma} \sum_{\text {clustercrystals }} G(\mathrm{GeV} / \mathrm{ADC}) \cdot C_{i} \cdot A_{i}
$$

where the sum is over the crystals in a cluster. $A_{i}$ are the reconstructed amplitudes in ADC counts, $C_{i}$ is the inter-calibration constant while $G$ is the ECAL energy scale. The factor $F_{e, \gamma}$ is defined as an additional energy correction which depends on the type of the particle, its energy and pseudorapidity and in particular takes into account shower leakage and bremsstrahlung losses for electrons.

The CMS ECAL was pre-calibrated prior to LHC collisions with an overall precision of $0.5 \%$ $2 \%$ in EB and 5\% in EE $[4,5]$, exploiting test beam measurements, laboratory measurements of the crystals light yield and photodetector gain, exposure to cosmic rays and muons from beam dumps. The global energy scale was set at test beams and verified using cosmic muons.

The calibration precision is improved in-situ using LHC collision data. Several strategies have been explored:

- the $\phi$-symmetry inter-calibration is a fast calibration method and is based on the invariance around the beam axis of energy flow in minimum bias events; it allows to inter-calibrate 
crystals in a ring at the same pseudorapidity. Inhomogeneities in the detector material limit the precision of the method to about $1.5 \%-3 \%$ depending on the channel pseudorapidity

- the $\pi^{0}$ and $\eta$ calibration exploits the mass peak of photon pairs selected as $\pi^{0}(\eta) \rightarrow \gamma \gamma$ candidates (Fig. 2); it is useful at the start-up also to investigate the ECAL energy scale

- isolated electrons from $W \rightarrow e v$ and $Z \rightarrow e^{+} e^{-}$decays can be used to compare the energy measured in ECAL to the track momentum measured in the silicon tracker; this will be the primary channel-to-channel calibration tool until several $\mathrm{fb}^{-1}$ of integrated luminosity will be collected

- di-electron resonances such as $J / \psi \rightarrow e^{+} e$ and $Z \rightarrow e^{+} e^{-}$can be used to monitor and correct the absolute ECAL energy scale.
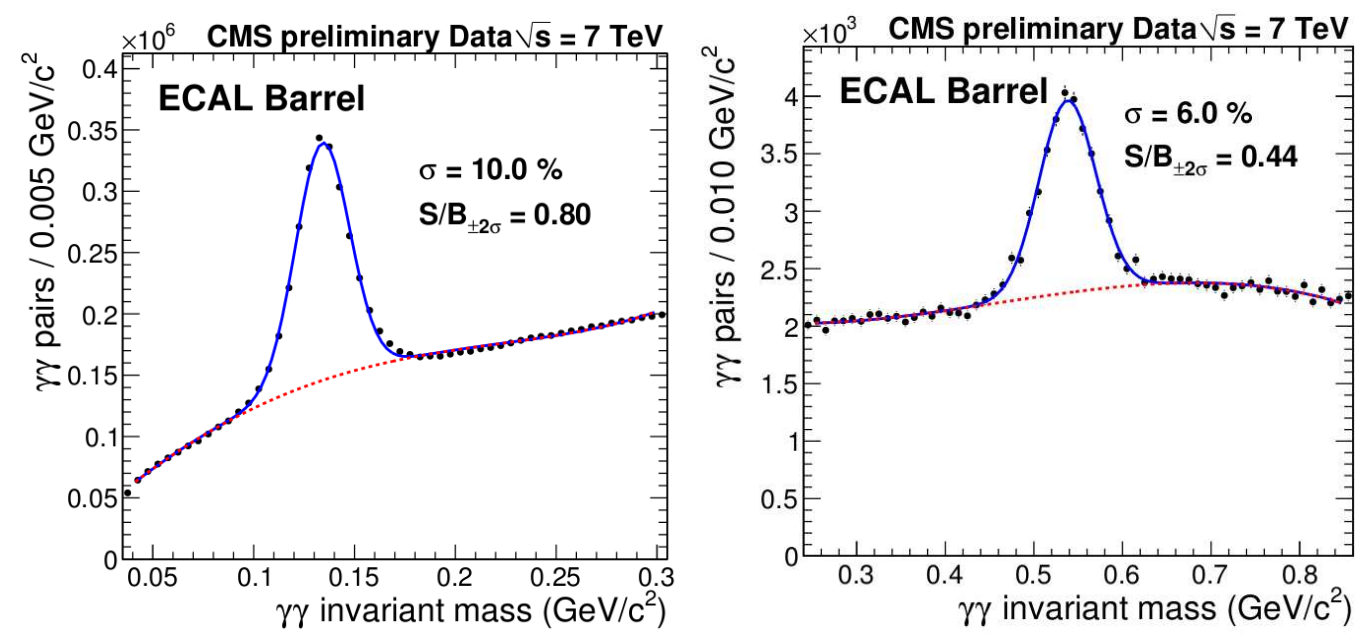

Figure 2: Invariant mass distribution from photon pairs selected as $\pi^{0}$ (left) and $\eta$ (right) candidates from 7 $\mathrm{TeV}$ collision data. The distributions are obtained for $0.31 \mathrm{nb}^{-1}$.

Figure 3 shows the inter-calibration precision as a function of the crystal $\eta$ index for three different methods ( $\phi$-symmetry, $\pi^{0} \rightarrow \gamma \gamma$, muons from beam dumps) and the one obtained from the combined measurements, resulting in a precision of $0.6 \%$ in the central region of the calorimeter with only $250 \mathrm{nb}^{-1}$ [6]. This accuracy is already close to the $0.5 \%$ required for $H \rightarrow \gamma \gamma$ discovery. The inter-calibration of the ECAL crystals will continue to improve with more LHC data.

The low mass di-photon resonances can also be exploited at the start-up to set the ECAL energy scale by comparing the peak position from data and from the MC simulation. The agreement has been found to be at the $1 \%(3 \%)$ level in $\mathrm{EB}(\mathrm{EE})$. On a longer term, other physics events such as $J / \psi \rightarrow e^{+} e^{-}, Z \rightarrow e^{+} e^{-}$(Fig. 4), $Z \rightarrow \mu \mu \gamma$, are used at this purpose.

\section{Summary}

The CMS ECAL status and performances with the first 2010 proton-proton LHC collisions have been presented. The ECAL stability is found to be well within specifications and is constantly 

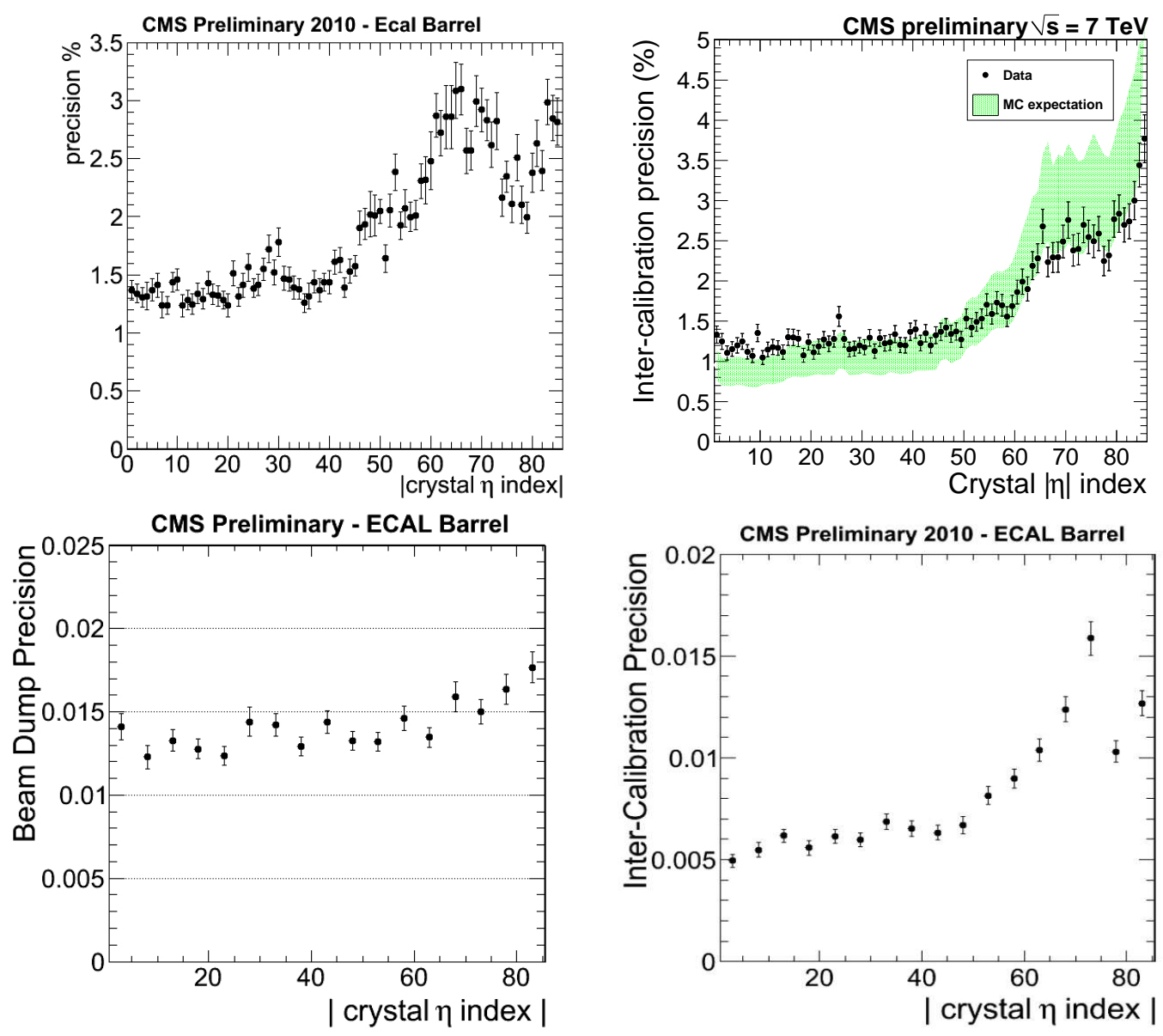

Figure 3: Inter-calibration precision as a function of the crystal $\eta$ index in EB using different strategies: $\phi$-symmetry (top-left), $\pi^{0} \rightarrow \gamma \gamma$ (top-right), muons from beam dumps (bottom-left), and the combined precision (bottom-right).

monitored. First collisions provided the opportunity to test the understanding of basic observables and to perform in-situ calibration with different methods. An inter-calibration precision of about $0.6 \%$ in the central barrel region has been achieved already with a modest integrated luminosity and the ECAL energy scale has been tested at the percent level.

\section{References}

[1] CMS Collaboration, The CMS experiment at the CERN LHC, JINST 0803 S08004 (2008)

[2] L. Evans and P. Bryant (eds.), LHC Machine, JINST, vol. 3, S08001, (2008)

[3] CMS Collaboration, Electromagnetic calorimeter commissioning and performance with $7 \mathrm{TeV}$ data, CMS NOTE 2010/12 (2010)

[4] P. Adzic et al., Intercalibration of the barrel electromagnetic calorimeter of the CMS experiment at start-up, JINST 3 (2008) P10007

[5] CMS Collaboration, Performance and operation of the CMS electromagnetic calorimeter, JINST 5 (2010) T03010. 


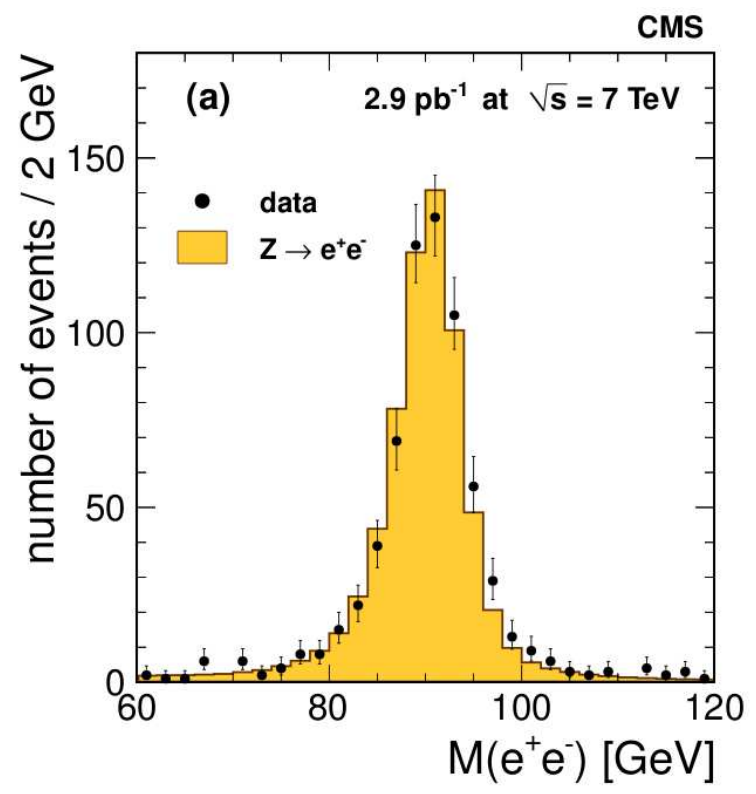

Figure 4: Di-electron mass spectrum reconstructed from $7 \mathrm{TeV}$ collision data (black dots) with an integrated luminosity of $2.9 \mathrm{pb}^{-1}$; the histogram represents the simulation [7].

[6] CMS Collaboration, Electromagnetic calorimeter calibration with $7 \mathrm{TeV}$ data, CMS Physics Analysis Summary EGM-10-003 (2010)

[7] CMS Collaboration, Measurements of Inclusive $W$ and $Z$ Cross Sections in pp Collisions at $\sqrt{s}=7$ $\mathrm{TeV}$, JHEP 01 (2011) 080, arXiv:1012.2466 